\title{
Harmonium: An Ultrafast Vacuum Ultraviolet Facility
}

\author{
Christopher A. Arrell ${ }^{\star a}$, José Ojedaa ${ }^{a}$ Luca Longettia, Alberto Crepaldib, Silvan Roth ${ }^{\mathrm{b}}$, Gianmarco \\ Gatti $^{b}$, Andrew Clarkc , Frank van Mourika ${ }^{a}$ Marcel Drabbels ${ }^{c}$, Marco Grioni ${ }^{b}$, and Majed Cherguia
}

\begin{abstract}
Harmonium is a vacuum ultraviolet (VUV) photon source built within the Lausanne Centre for Ultrafast Science (LACUS). Utilising high harmonic generation, photons from 20-110 eV are available to conduct steadystate or ultrafast photoelectron and photoion spectroscopies (PES and PIS). A pulse preserving monochromator provides either high energy resolution $(70 \mathrm{meV}$ ) or high temporal resolution (40 fs). Three endstations have been commissioned for: a) PES of liquids; b) angular resolved PES (ARPES) of solids and; c) coincidence PES and PIS of gas phase molecules or clusters. The source has several key advantages: high repetition rate (up to $15 \mathrm{kHz}$ ) and high photon flux ( $10^{11}$ photons per second at $38 \mathrm{eV}$ ). The capabilities of the facility complement the Swiss ultrafast and X-ray community (SwissFEL, SLS, NCCR MUST, etc.) helping to maintain Switzerland's leading role in ultrafast science in the world.
\end{abstract}

Keywords: Photoelectron spectroscopy · Photoion spectroscopy $\cdot$ Ultrafast science

\section{Introduction}

During the last decades the abilities of ultrafast science have increased dramatically. Early techniques, while still providing a plethora of information to physicists and chemists, are often unable to follow structural dynamics as they remain sensitive to valence electrons which are usually delocalized over the molecule. Ultrafast techniques using higher photon energies have been developed using synchrotron sources, ${ }^{[1]}$ free electron lasers ${ }^{[2]}$ and lab-based lasers. ${ }^{[3]}$ These advanced techniques have allowed ultrafast structural dynamics to be followed by accessing inner-shell and core level electrons, but are limited by either: temporal resolution, beam-time access or choice of photon energies (and resolution) available. To increase the range of tools available to scientists, the Harmonium ultrafast vacuum ultraviolet facility has been constructed within the Lausanne Centre for Ultrafast Science (LACUS) at EPFL.

The standard technique for ultrafast measurements is pump-probe where a pump pulse perturbs the system and a probe pulse follows the evolution of the system back to equilibrium. The photon energies of Harmonium are ideal for photoelectron and photoion spectroscopy (PES and PIS) and as such three endstations have been constructed for PES of liquids, and solids called ASTRA, and of clusters and helium droplets (CD) (Fig. 1). PES has the advantage of directly mapping the electron states of a system and is not blind to 'dark states' as can be the case for all-optical spectroscopies. Furthermore, with sufficiently high photon energies, inner-shell and core levels are accessible giving access to element specific bands and hence chemical markers of a system.
The Harmonium facility is a large source built over the past five years. It incorporates three amplified laser systems, a VUV source and three endstations. It is over twenty metres long and involves four different research groups from the chemistry and physics institutes of EPFL. A schematic of the system is given in Fig. 2 and a description of the photon source and capabilities of the endstations is given in the following sections.

\section{Light Source}

\subsection{Vacuum Ultraviolet Probe}

As Harmonium has multiple users, various probe configurations are required. For example a high temporal resolution (sub$50 \mathrm{fs}$ ) is required to follow the dynamics of molecular systems, high energy resolution (sub-100 meV) to follow electronic band
${ }^{*}$ Correspondence: Dr. C. A. Arrell E-mail: christopher.arrell@epfl.ch

aLaboratory of Ultrafast Spectroscopy and Lausanne Centre for Ultrafast Science (LACUS)

ISIC Station 6

Ecole Polytechnique Fédérale de Lausanne (EPFL) $\mathrm{CH}-1015$ Lausanne

'Laboratory for Electron Spectroscopy and Lausanne Centre for Ultrafast Science (LACUS)

ICMP, Station 3

EPFL, CH-1015 Lausanne

cLaboratory for Molecular Nanodynamics and Lausanne Centre for Ultrafast Science (LACUS) ISIC, Station 6

EPFL, CH-1015 Lausanne

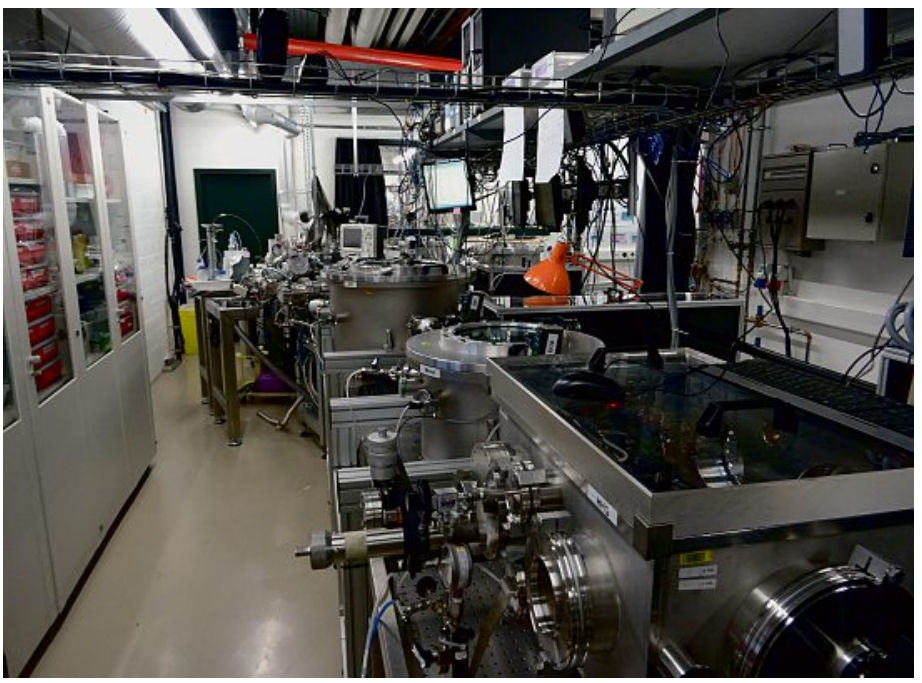

Fig. 1. View of the VUV source chambers of Harmonium. Behind the photographer are the laser sources, to the far left of the picture the Liquid endstation and behind the lab wall the Solid and CD endstations. 


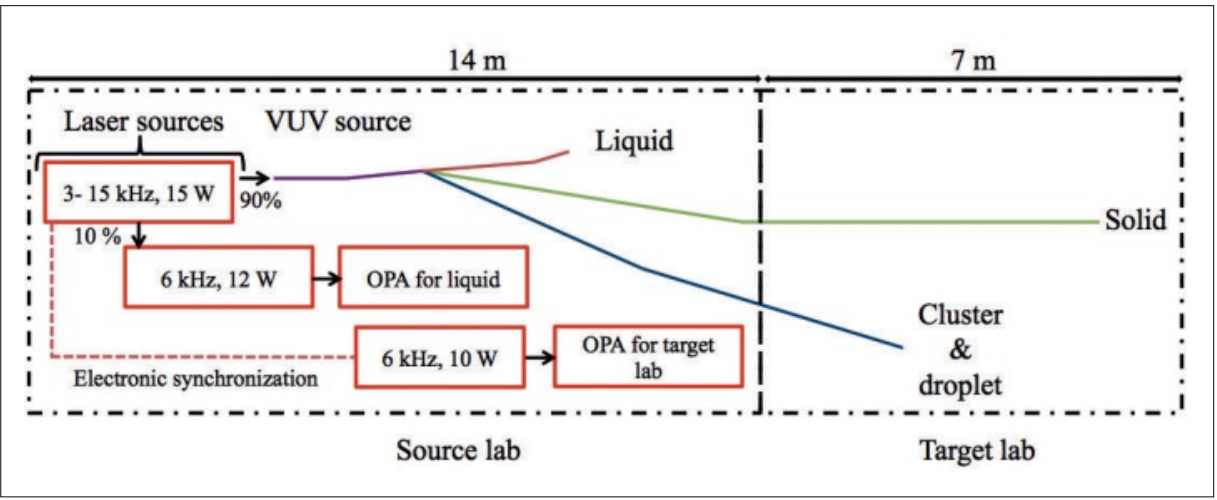

Fig. 2. Schematic of the Harmonium facility. The laser systems are shown in red, the purple line represents the VUV source and monochromatisation, the orange line the Liquid endstation, the green the Solid endstation and the blue the CD endstation. The beam path of the lasers is omitted for clarity.

dispersion, a high photon (100 eV) energy to access Auger states or a photon energy below $21 \mathrm{eV}$ to avoid ionisation from helium.

By using an intense laser pulse to tunnel ionise a target (typically a noble gas) high harmonic generation (HHG) produces a spectrum extending into the VUV. HHG intrinsically yields a spectrum of harmonics (Fig. 3) extending over many 10 's of eV with a short pulse structure, ${ }^{[4]}$ which requires subsequent monochromatisation for PES.

To maintain flexibility, a grating-based monochromator design was chosen in collaboration with CNR-IFN in Padova. The design, with the grooves running parallel to the VUV propagation direction, limits the temporal broadening introduced by a path length difference from the grating. In so doing and by using a collimated VUV beam, the number of grooves illuminated is controlled and hence also the temporal broadening of the pulse. This technique is reviewed in ref. [5].

To provide either high energy ( 70 meV) or high temporal resolution $(\sim 40$ fs) over the full energy range accessible by Harmonium (30-110 eV) one of four different gratings with different groove densities can be used. The performance of the monochromator is shown in Fig. 4 and a full report of the system is given in ref. [6]. For photon energies up to $40 \mathrm{eV}$, argon was used as the generation medium in the HHG process and neon was used for energies up to $110 \mathrm{eV}$, typically providing $1 \times$ $10^{11}$ photons/second (measured at Liquid endstation target) at 30-40 eV and $2 \times 10^{8}$ photons/second (measured at Liquid endstation target) between 50-100 eV.

The optics of the monochromator images the effective entrance slit of the instrument to the exit slit in a 1:1 configuration. As it is not feasible to use an entrance slit as this would be close to the fundamental IR focus, the VUV source spot is used which is imaged to a variable exit slit of the monochromater. Typically the VUV source diameter is $100 \mu \mathrm{m}$ FWHM, but is however highly dependent upon the focal parameters of the fundamental laser and phase matching. The control of the VUV source spot size including the divergence of the VUV gives further control over the temporal response of the system and will be the subject of a future publication.

The VUV focal requirements differ for the Liquid endstation as compared to those required for ASTRA and the CD endstations. For the former, due to the small diameter $(\sim 25 \mu \mathrm{m})$ of the liquid jet it is

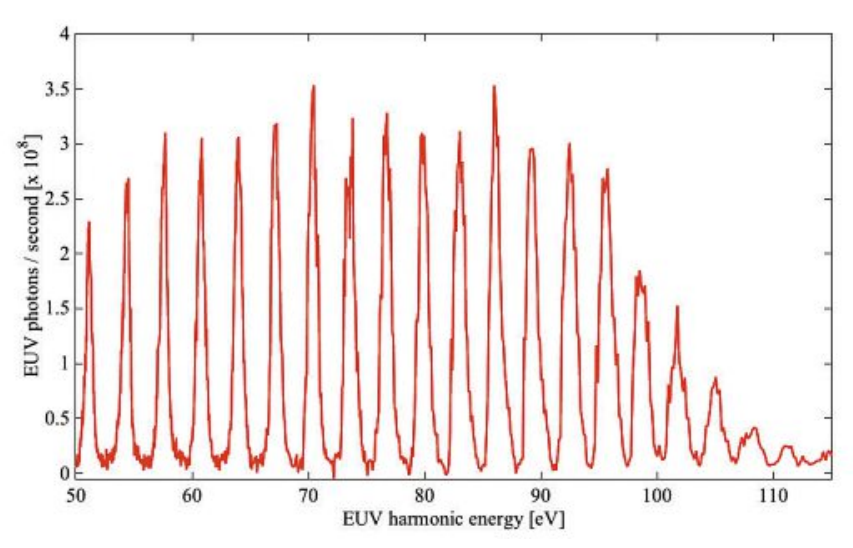

Fig. 3. A spectrum of VUV produced by Harmonium. The $\mathrm{HHG}$ yields a spectrum that required monochromatisation. preferential to have a similarly sized focus to reduce photoelectron contributions from gas molecules (see section 3.3), while a larger focal spot is desired for the ARPES and $C D$ to reduce space charge effects and to better match the collection volume for their respective detectors. A gold-coated ellipsoidal mirror (Zeiss AG) is used for the Liquid phase endstation to reduce the $\sim 100$ $\mu \mathrm{m}$ source size to $\sim 25 \mu \mathrm{m}$ with a 2000 $\mathrm{mm}$ entrance arm and a $500 \mathrm{~mm}$ exit arm (6.0 $0^{\circ}$ angle of incidence). For the ASTRA and $\mathrm{CD}$ endstations toroidal mirrors are used in a $2 \mathrm{f}-2 \mathrm{f}$ configuration, providing $\mathrm{a} \sim 100 \mu \mathrm{m}$ focus. Given the large endstation footprint, the entrance/exit arm of the mirror for ASTRA is $5100 \mathrm{~mm}\left(4.5^{\circ}\right.$ angle of incidence) and $3900 \mathrm{~mm}\left(4.0^{\circ}\right.$ angle of incidence) for the $\mathrm{CD}$ mirror. To ensure the full beam is collected the carbon-coated mirrors (Pilz Optics) dimensions are $440 \times$ $60 \times 50 \mathrm{~mm}^{3}$. Pointing control of the VUV spot to accurately position and optimise the respective foci is critical. To achieve this the ellipsoidal mirror uses a six-axis hexapod positioner (SpaceFab, PI GmbH) while the toroidal mirrors have actuators on the three principle mirror axes (Alca Technology Srl). Precise alignment of the VUV focus is achieved in all cases by imaging the VUV focus on a YAG:Ce crystal.

\subsection{Optical Pump}

To minimise disruption when switching between endstations and given the different temporal/energy resolution requirements of the optical pump laser systems, two separated laser systems have been installed to provide the optical pump.

For the Liquid station where typically higher temporal resolution is required ( $<50 \mathrm{fs}$ ) and a pump in the UV to visible range is desirable, a booster stage has been constructed for the principal laser system driving the HHG. A fraction $(\sim 10 \%)$ of the uncompressed $6 \mathrm{kHz}$ output of the regenerative amplifier is subsequently double passed in a home-built cryogenically cooled Ti-Sapphire multi-pass amplifier to provide $12 \mathrm{~W}$ at $6 \mathrm{kHz}$ to pump an optical parametric amplifier (OPA) dedicated to the Liquid endstation.

A separate regenerative amplifier (Coherent Astrella) producing $6 \mathrm{~W}$ at 6 $\mathrm{kHz}$, pumps an OPA providing the optical pump (200 nm - $12 \mu \mathrm{m})$ for either ASTRA or the CD endstation. This oscillator of the Astrella is electronically synchronised to the oscillator seeding the principal regenerative amplifier of the system. There is typically a sub-100 fs jitter in-between the two outputs, which is acceptable for the trARPRES and CD measurements. Furthermore provision is provided to pump with the intrinsically synchronised pump arm for the Liquid endstation if required. 


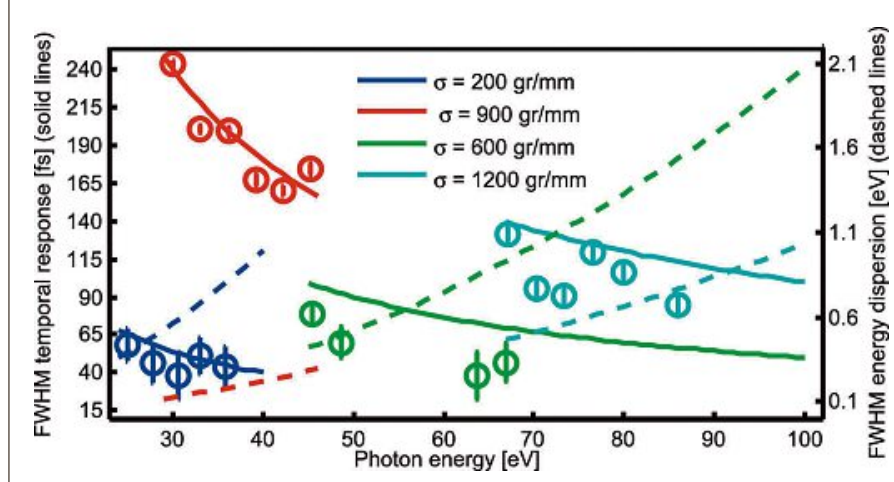

Fig. 4. Grating response of the Harmonium monochromator Depending on the selected grating (and hence groove density) either high temporal resolution or high energy resolution is provided. Figure from ref. [6].

\section{Endstations}

\subsection{Liquid Endstation}

For many photochemistry processes the solvent has a large effect on the energetics and temporal outcomes of photoexcitation. Therefore, to fully apply time-resolved techniques to chemistry, these techniques need to be implemented in solution. For these reasons we have developed an endstation at Harmonium to conduct time-resolved liquid photoelectron spectroscopy (trLPES).

LPES was first introduced by Siegbahn and co-workers in $1973,{ }^{[7]}$ but it was not until the development of the liquid microjet technology by Faubel and co-workers in $1988^{[8]}$ that the technique became more widely implemented. The Faubel design uses a small glass capillary $(20 \mu \mathrm{m}$ in diameter) to inject a continuous flow of liquid in vacuum. When the velocity is sufficiently high $\left(30 \mathrm{~ms}^{-1}\right)$ a region of laminar flow is formed extending for several millimetres. This region provides a liquidvacuum interface where photoelectrons can be directly collected from the liquid. This technique combined with cryo-pumping and a specialist electron spectrometer design ${ }^{[9]}$ allows photoelectron spectra to be collected from a range of different solvents and respective solutes. The LPES spectrum of $\mathrm{NaI}$ in an aqueous solution is shown in Fig. 5, along with a pure water spectrum. Due to electrokinetic charging it is not possible to measure a PES from pure water and a small concentration of salt is added, here $25 \mathrm{mM} \mathrm{NaCl}$, see ref. [10] for more details. This data, taken at $83 \mathrm{eV}$, exploits the high photon energy available from Harmonium to access the inner-shell I 4d shell, a first for a VUV source based on HHG. The spectra contain contributions from both the liquid jet and surrounding evaporated gas. The relative binding energies of gas and liquid phase are separated by $\sim 1.5 \mathrm{eV}$ allowing the gas contributions to be subtracted if required. Examples of this can be found in ref. [11].

Work is currently underway at Harmonium to utilize this ability to access the inner shell to study both the electronic and structure dynamics following charge transfer by measuring both the valence and inner-shell dynamics.

Recent work at the Liquid endstation has focused on several areas. As LPES is a relatively new technique, ${ }^{[12]}$ methods for trLPES are still under development. ${ }^{[9,13-15]}$ Importantly to aid the progress we have recently identified and characterized the laser-assisted photoelectric effect (LAPE) from liquids, a strong field process occurring within the cross-correlation of the pump and probe fields. ${ }^{[16]}$ Following this development, we have been able to utilise the high temporal resolution to follow the sub-100 fs reduction dynamics of $\left[\mathrm{Fe}(\mathrm{CN})_{6}\right]^{3-}$ following a photoinduced ligand-to-metal charge transfer (LMCT).

An important feature of Harmonium that contrasts with higher photon energy sources (e.g. synchrotrons and free electron lasers) is that LPES at Harmonium is sensitive to the surface of the liquid jet. Owing to the photon energy range of Harmonium $(20-110 \mathrm{eV})$ the inelastic mean free path of the photoelectron gives sensitivity to the surface or near surface in contrast to the bulk sensitivity of UPS and XPS. The significance is clear, interfacial chemistry is energy of $83 \mathrm{eV}$. key to understanding biological processes and Harmonium is an ideal tool to advance this research.

\subsection{Cluster and Droplet Endstation}

The evolution of molecular systems is governed by the potential energy landscape describing the interactions between its constituents and the dynamics taking place on this potential energy landscape. For isolated molecules, both aspects can nowadays be investigated in great detail and precision. The situation is much more complex for molecules in solution, due to environmental interactions. Studying the effect of microsolvation can form a link between isolated systems and solution phase samples, as it allows for a systematic separation of environmental interactions from intrinsic sample effects.

An endstation for microsolvation studies based on time-resolved photoelectron spectroscopy (trPES) is currently under construction at Harmonium (Fig. 6). Size-controlled clusters or nanodroplets containing a single solute molecule are formed using a combination of molecular beam and pickup techniques. The energy redistribution in the system after photoexcitation of the solute will be explored using trPES. The VUV radiation provided by Harmonium allows probing of energy levels all the way down to the ground state of the system. The detection system of the setup is based on a double imaging photoelectron photoion coincidence (i2-PEPICO) spectrometer. Upon photoionisation of an analyte in a time-resolved pump-probe process, photoelectrons and photoions are extracted in opposite directions by electric fields in a double velocity map imaging (VMI) configuration and detected in coincidence on two-dimensional spatial detectors with a high time resolution. The i2-PEPICO technique ${ }^{[17-19]}$ allows for an extremely detailed, multi-

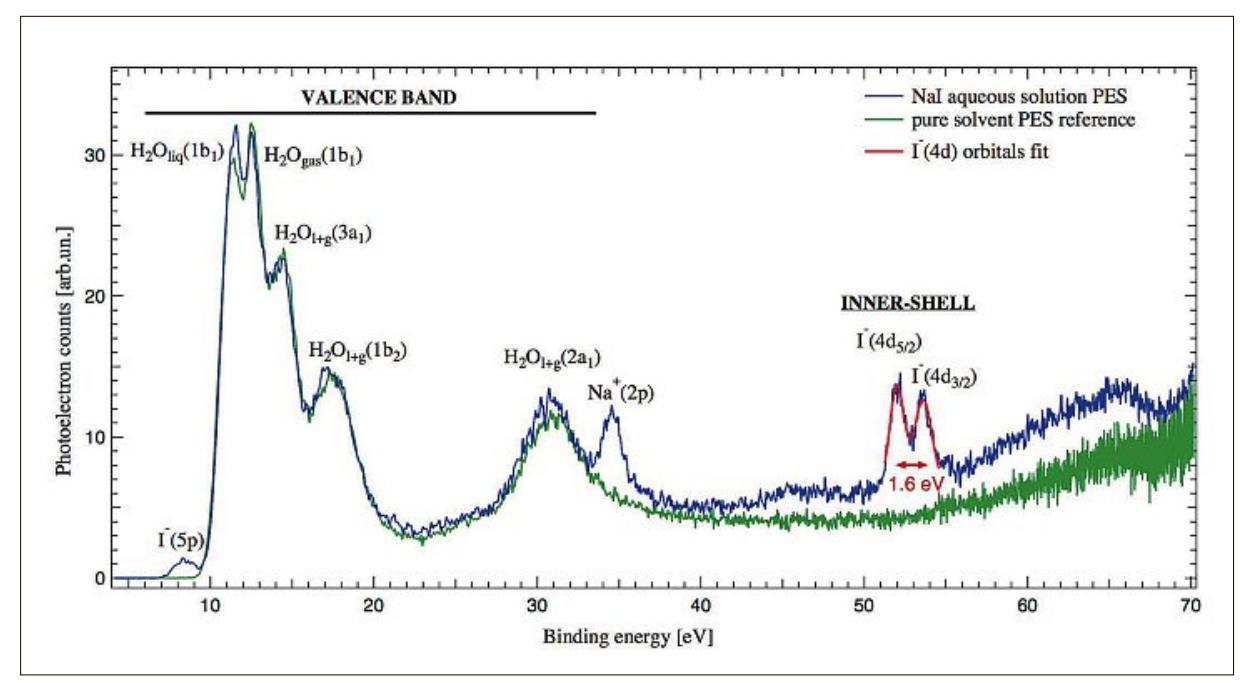

Fig. 5. Photoelectron spectra of water and an aqueous solution of Nal. Measured with photon 
plexed view into excited state dynamical processes. Photoinduced fragmentations can be readily identified by time-of-flight measurements and, by recording electrons and ion fragments in coincidence, photoelectron spectra for each photofragment can be recorded. Coincidence detection also gives angular distributions of both photoelectrons and photofragments, allowing near-laboratory frame photoelectron angular distributions to be recorded, ${ }^{[20]}$ which serve as an extremely effective probe of the symmetry of the electronic states being ionized. [21] Utilizing each of these various sources of information in 2-PEPICO makes it possible to disentangle different contributions to the dynamics, a necessity when considering systems with multiple relaxation channels. ${ }^{[22]}$

A prototypical example for such studies is the DNA base adenine. Experimental and theoretical studies on gas-phase adenine have identified several efficient non-radiative decay processes proceeding via conical intersections ${ }^{[23-25]}$ that allow for a fast conversion of the electronic energy to heat. It is hypothesized that this influences adenine's biological role as a DNA base by serving as a protective mechanism for DNA from solar UV light. However, there is still debate whether the same behaviour is exhibited once adenine is hydrogen-bonded and base-stacked in solution with DNA. ${ }^{[26,27]}$ Previous studies showed a marked decrease in the ${ }^{1} L_{b}\left(\pi \pi^{*}\right)$ excited electronic state lifetime induced by a superfluid helium solvent.[28] This was tentatively attributed to a lowering of the potential energy barrier blocking access to a conical intersection. The degree of change is significant given that superfluid helium is ostensibly an extremely non-perturbative solvent. Aqueous environments would induce markedly larger changes, so much so that individual contributions are prone to be washed out in bulk phase. Controlling solvation by se- lecting cluster/droplet sizes will allow a clear picture of solvent-induced dynamics to be established.

\subsection{Solid Phase Endstation}

A detailed description of the solid phase endstation can be found in the article 'Time-resolved ARPES at LACUS: Band Structure and Ultrafast Electron Dynamics of Solids' in the same issue of this journal,[29] a summary is presented in the following. Angular Resolved Photoelectron Spectroscopy (ARPES) is a powerful tool to investigate the electronic structure of a solid. ${ }^{[30]}$ Making use of the photoelectric effect, electrons are emitted from the illuminated sample surface into vacuum. The conservation of energy and momentum parallel to the surface during the emission process allows us to trace back the electron to its initial state inside the solid. Combining the ASTRA ARPES endstation with the capabilities of Harmonium to probe ultrafast phenomena provides new insight into the electronic bandstructure, which are not possible with conventional photoelectron spectroscopy. By means of pump-probe techniques, electrons can be excited (pumped) from below the Fermi level into the unoccupied part of band-structure, and their subsequent relaxation followed by a VUV probe. Other than two-photon photoemission experiments where the probe consists of two times the frequency doubled or tripled fundamental $(2 \times 3.1 \mathrm{eV}$ or $2 \times 4.6 \mathrm{eV})$, HHG photons up to several tens of $\mathrm{eV}$, allow us to probe larger areas of the $\mathrm{k}$-space e.g. the entire Brillouin zone of a material. Typically for such processes a high energy resolution (below $100 \mathrm{meV}$ ) and a good momentum resolution (better than $0.1 \AA^{-1}$ ) is required. Harmonium is optimised for these requirements with the variable grating configuration of the monochromator providing $\sim 75$ meV energy resolution and the focusing parameters combined with the ARPES

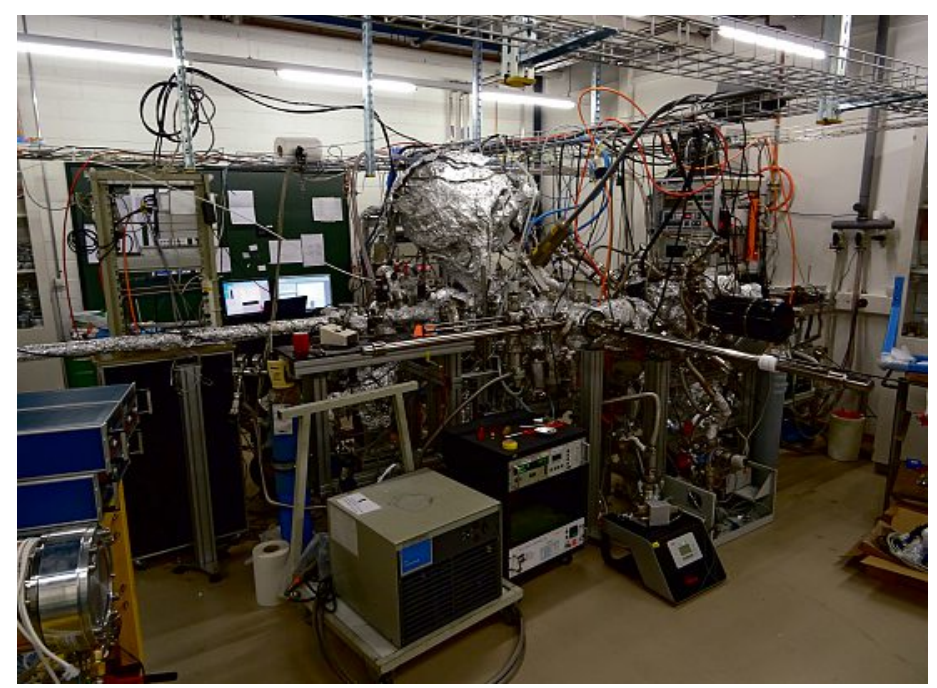

spectrometer providing $0.05 \AA^{-1}$ momentum resolution.

Fig. 7 summarizes a time-resolved ARPES (trARPES) study of $\mathrm{Bi}_{2} \mathrm{Se}_{3}$, a layered compound from the family of topological insulators. ${ }^{[31,32]}$ The stacking of the layers is visualized in Fig. 7a. These materials are promising candidates for future nanotechnology applications such as quantum computing, ${ }^{[33]}$ spintronics, ${ }^{[34,35]}$ high performance field effect transistors ${ }^{[36]}$ and flexible electrodes in solar cells. ${ }^{[37]}$ These effects rely on the excitation of electrons from the ground state into unoccupied part of the materials bandstructure.

Therefore, Harmonium with its ARPES endstation is ideal to follow the dynamics of such excitation. The curve shown in Fig. 7 shows the population dynamics of the electronic states above the Fermi level $\left(E_{F}\right)$ following an optical pump (800 nm). The ARPES spectrum shows the conduction band minimum slightly below the $\mathrm{E}_{\mathrm{F}}$, while the top of the valance band is at $-0.4 \mathrm{eV}$. In between the two bands is the topologically protected surface state. The first spectrum shows the bandstructure at the point of the highest excitation. With increasing time between the pump and the probe pulse, relaxation processes lead to a continuous relaxation of the states populated above $\mathrm{E}_{\mathrm{F}}$. The second and the third image show the bandstructure 4 ps and 8 ps after the maximum excitation. With the addition of the dedicated optical pumping arm for the ASTRA and CD endstations providing a pump between $200 \mathrm{~nm}$ $12 \mu \mathrm{m}$ inter-band transitions can be excited resonantly and studied.

\section{Conclusions}

We believe the Harmonium facility represents a significant benefit to the ultrafast community within Switzerland. The range of endstations covers samples from gas phase molecules through to clusters, liquids and solids providing a level of functionality more commonly found at a national level such as Artemis in the UK. ${ }^{[38]}$ The ASTRA endstation also has the capability to probe molecules on surfaces. Through our associate membership of LaserLab and collaborative networks within Switzerland (e.g. NCCR MUST) the source is also available for external users, either to use the current endstations or to bring their own experiment. With the current opening of SwissFEL, Harmonium is operational at an opportune moment to act as a testing ground and complementary source to the larger ultrafast facilities where beamtime access is limited. 


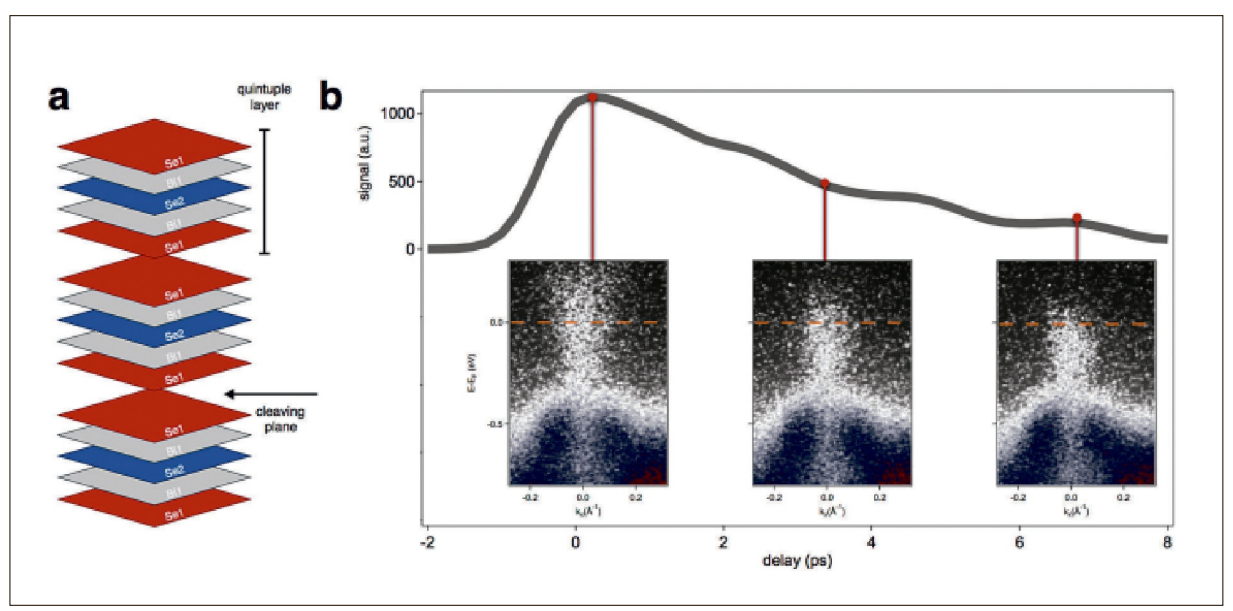

Fig. 7. Time-resolved ARPES study of $\mathrm{Bi}_{2} \mathrm{Se}_{3}$ measured at Harmonium.

11] C. J. Milne, T. J. Penfold, M. Chergui, Coord. Chem. Rev. 2014, 277, 44.

[2] J. Feldhaus, J. Phys. B At. Mol. Opt. 2010, 43, 194002.

[3] M. Drescher, M. Hentschel, R. Kienberger, M. Uiberacker, V. Yakovlev, A. Scrinzi, T. Westerwalbesloh, U. Kleineberg, U. Heinzmann, F. Krausz, Nature 2002, 419, 803.

[4] F. Frank, C. Arrell, T. Witting, W. A. Okell, J. McKenna, J. S. Robinson, C. A. Haworth, D. Austin, H. Teng, I. A. Walmsley, J. P. Marangos, J. W. G. Tisch, Rev. Sci. Instrum. 2012, 83 071101.

[5] F. Frassetto, N. Fabris, P. Miotti, L. Poletto, Photonics 2017, 4, 14

[6] J. Ojeda, C. A. Arrell, J. Grilj, F. Frassetto, L. Mewes, H. Zhang, F. van Mourik, L. Poletto, M. Chergui, Struct. Dyn. 2016, 3, 023602.

[7] H. Siegbahn, K. Siegbahn, J. Electron. Spectrosc. 1973, 2, 319.

[8] M. Faubel, S. Schlemmer, J. P. Toennies, Z. Phys. D Atom. Mol. Cl. 1988, 10, 269.

[9] C. A. Arrell, J. Ojeda, M. Sabbar, W. A. Okell, T. Witting, T. Siegel, Z. Diveki, S. Hutchinson, L. Gallmann, U. Keller, F. van Mourik, R. T. Chapman, C. Cacho, N. Rodrigues, I. C. E. Turcu, J. W. G. Tisch, E. Springate, J. P. Marangos, M. Chergui, Rev. Sci. Instrum. 2014, $85,103117$.

[10] N. Preissler, F. Buchner, T. Schultz, A. Lubcke, J. Phys. Chem. B 2013, 117, 2422.

[11] B. Winter, R. Weber, W. Widdra, M. Dittmar, M. Faubel, I. V. Hertel, J. Phys. Chem. A 2004, 108 , 2625.

[12] B. Winter, M. Faubel, Chem. Rev. 2006, 106, 1176.

[13] I. Jordan, M. Huppert, M. A. Brown, J. A. van
Bokhoven, H. J. Worner, Rev. Sci. Instrum. 2015, 86, 123905 .

[14] M. Borgwardt, M. Wilke, I. Y. Kiyan, E. F. Aziz, Phys. Chem. Chem. Phys. 2016, 18, 28893. 2017, 4, 044014.

[16] C. A. Arrell, J. Ojeda, L. Mewes, J. Grilj, F. Frassetto, L. Poletto, F. van Mourik, M. Chergui, Phys. Rev. Lett. 2016, 117, 143001.

[17] A. Vredenborg, W. G. Roeterdink, M. H. M. Janssen, Rev. Sci. Instrum. 2008, 79, 063108.

[18] A. Bodi, P. Hemberger, T. Gerber, B. Sztaray, Rev. Sci. Instrum. 2012, 83, 083105.

[19] G. A. Garcia, B. K. C. de Miranda, M. Tia, S. Daly, L. Nahon, Rev. Sci. Instrum. 2013, 84, 053112.

[20] O. Gessner, A. M. D. Lee, J. P. Shaffer, H. Reisler, S. V. Levchenko, A. I. Krylov, J. G. Underwood, H. Shi, A. L. L. East, D. M. Wardlaw, E. T. H. Chrysostom, C. C. Hayden, A. Stolow, Science 2006, 311, 219.

[21] K. L. Reid, Ann. Rev. Phys. Chem. 2003, 54, 397.

[22] P. Maierhofer, M. Bainschab, B. Thaler, P. Heim, W. E. Ernst, M. Koch, J. Phys. Chem. A 2016, 120, 6418.

[23] L. Blancafort, J. Am. Chem. Soc. 2006, 128, 210.

[24] C. Plutzer, K. Kleinermanns, Phys. Chem. Chem. Phys. 2002, 4, 4877.

[25] C. M. Marian, J. Chem. Phys. 2005, 122, 104314.

[26] J. Luo, Y. Liu, S. Yang, J. Photochem. Photobio. A 2017, 337, 1 .

[27] N. K. Schwalb, F. Temps, Science 2008, 322, 243.

[28] S. Smolarek, A. M. Rijs, W. J. Buma, M. Drabbels, Phys. Chem. Chem. Phys. 2010, 12, 15600.

[29] A. Crepaldi, S. Roth, G. Gatti, C. A. Arrell, J. Ojeda, F. van Mourik, P. Bugnon, A. Magrez, H.
[15] J. Nishitani, C. W. West, T. Suzuki, Struct. Dyn.
Berger, M. Chergui, M. Grioni, Chimia 2017, $71,273$.

[30] S. Hüfner, 'Photoelectron Spectroscopy', Springer, 2003.

[31] Y. Xia, D. Qian, D. Hseih, L. Wray, A. Pal, H. Lin, A. Bansil, D. Grauer, Y. S. Hor, R. J. Cava, M. Z. Hasan, Nat. Phys. 2009, 5, 398.

[32] H. J. Zhang, C.-X. Liu, X.-L. Qi, X. Dai, Z. Fang, S.-C. Zhang, Nat. Phys. 2009, 5, 438.

[33] M. Z. Hasan, C. L. Kane, Rev. Mod. Phys. 2010, 82,3045 .

[34] X. B. Xiao, S. Y. A. Yang, Z. F. Liu, H. L. Li, G. H. Zhou, Sci. Rep. 2015, 5, 7898.

[35] Y. Cao, J. A. Waugh, X.-W. Zhang, J.-W. Luo, Q. Wang, T. J. Reber, S. K. Mo, Z. Wu, A. Yang, J. Schneeloch, G. D. Gu, M. Brahlek, N. Bansal, S. Oh, A. Zunger, D. S. Dessau, Nat. Phys. 2013, 9, 499.

[36] H. Zhu, C. A. Richter, E. Zhao, J. E. Bonevich, W. A. Kimes, H.-J. Jang, H. Yuan, H. Li, A. Arab, O. Kirillov, J. E. Maslar, D. E. Ioannou, Q. Li, Sci. Rep. 2013, 3, 1757.

[37] H. Peng, W. Dang, J. Cao, Y. Chen, D. Wu, W. Zheng, H. Li, Z. X. Shen, Z. Liu, Nat. Chem. 2012, 4, 281.

[38] I. C. E. Turcu, E. Springate, C. A. Froud, C. M. Cacho, J. L. Collier, W. A. Bryan, G. R. A. J. Nemeth, J. P. Marangos, J. W. G.Tisch, R. Torres, T. Siegel, L. Brugnera, J. G. Underwood, I. Procino, W. R. Newell, C. Altucci, R. Velotta, R. B. King, J. D. Alexander, C. R. Calvert, O. Kelly, J. B. Greenwood, I. D. Williams, A. Cavalleri, J. C. Petersen, N. Dean, S. S. Dhesi, L. Poletto, P. Villoresi, F. Frassetto, S. Bonora, M. D. Roper, Proc. Spie. 2010, 7469, 746902. 